reported as estimated sample means with a 95\% confidence interval.

Results In our multi-center cohort, aneurysm occlusion was achieved in $88 \%(7 / 8)$ of treated aneurysms and clinical complications occurred in $0 \%(0 / 6)$ of patients. An illustrative example from our cohort is provided in figure 1 . In our meta-analysis of 46 aneurysms from 7 studies, the rate of aneurysm occlusion was $81 \%$ (95\% CI, 69\%-92\%) and the rate of clinical complications was 10\% (95\% CI, 2\%-18\%).

Conclusions Pipeline embolization of cerebral aneurysms of the M2-M4 segments of the MCA was reasonably effective and safe in a small group of selected patients. Further study is needed to validate these results.

Disclosures D. Lauzier: None. B. Root: None. Y. Kayan: 2; C; Microvention, Penumbra, Medtronic. J. Delgado Almandoz: 2; C; Medtronic, Microvention. J. Osbun: 2; C; Medtronic, Microvention. A. Chatterjee: None. K. Whaley: None. M. Tipps: None. C. Moran: 2; C; Medtronic, Cerenovus. A. Kansagra: 2; C; Penumbra, Microvention, iSchemaView.

\section{E-078 PIPELINE EMBOLIZATION OF PROXIMAL MIDDLE CEREBRAL ARTERY ANEURYSMS: A MULTICENTER COHORT STUDY}

${ }^{1} \mathrm{D}$ Lauzier*, ${ }^{1} \mathrm{~B}$ Root, ${ }^{2}{ }^{2}$ Kayan, ${ }^{2} \mathrm{~J}$ Delgado Almandoz, ${ }^{3} \mathrm{~J}$ Osbun, ${ }^{3} \mathrm{~A}$ Chatterjee, ${ }^{2} \mathrm{~K}$ Whaley, ${ }^{2} \mathrm{M}$ Tipps, ${ }^{4} \mathrm{C}$ Moran, ${ }^{3} \mathrm{~A}$ Kansagra. ${ }^{1}$ Mallinckrodt Institute of Radiology, Washington University School of Medicine, St. Louis, MO; ${ }^{2}$ Abbott Northwestern Hospital, Minneapolis, $M N{ }^{3}$ Mallinckrodt Institute of Radiology, Department of Neurological Surgery, Department of Neurology, Washington University School of Medicine, St. Louis, MO; ${ }^{4}$ Mallinckrodt Institute of Radiology, Department of Neurological Surgery, Washington University School of Medicine, St. Louis, MO

\subsection{6/neurintsurg-2021-SNIS.173}

Introduction/Purpose Flow diversion of aneurysms located in the M1 segment and middle cerebral artery bifurcation with Pipeline embolization device is sometimes performed, but further study is needed to support its regular use in aneurysm treatment. Here, we report measures of safety and efficacy for Pipeline embolization in the proximal middle cerebral artery in a multi-center cohort.

Materials and Methods Clinical and angiographic data of eligible patients undergoing Pipeline embolization of aneurysms

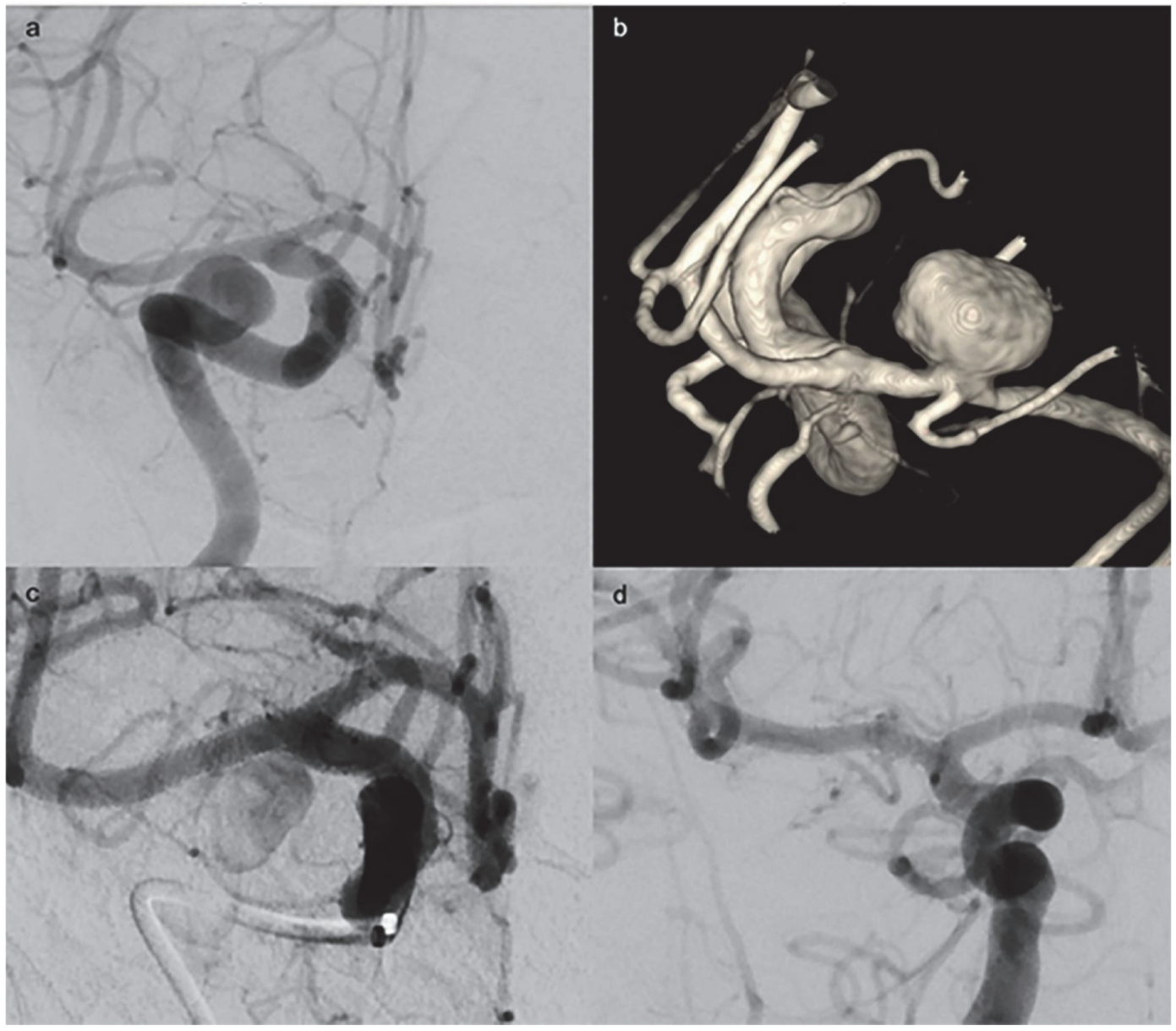

Abstract E-078 Figure 1 (a) Pre-treatment angiography and (b) pre-treatment 3D reconstruction show a $13.4 \times 12.0 \mathrm{~mm}$ aneurysm of the right M1 segment. (c) Immediate post-treatment angiography following deployment of $3.25 \times 14 \mathrm{~mm}$ PED showing contrast stasis within the aneurysm. (d) 32 month follow-up angiography demonstrates complete occlusion of the aneurysm 
located in the M1 segment and middle cerebral artery bifurcation were retrospectively obtained from participating centers and assessed for key clinical, angiographic, and cross-sectional imaging outcomes. Additional details were extracted for patients with complications.

Results In our multi-center cohort, complete aneurysm occlusion was achieved in $71 \%(17 / 24)$ of treated aneurysms. There were no deaths or disabling strokes, but non-disabling ischemic strokes occurred in $8 \%(2 / 24)$ of patients. For aneurysms in the M1 segment, complete aneurysm occlusion was observed in $75 \%(12 / 16)$ of aneurysms, aneurysm volume reduction was observed in $100 \%(16 / 16)$ of aneurysms, and non-disabling ischemic strokes occurred in $13 \%(2 / 16)$ of patients. An illustrative example of an M1 aneurysm treated with PED is provided in figure 1. For aneurysms at the middle cerebral artery bifurcation, complete aneurysm occlusion was observed in $63 \%(5 / 8)$ of aneurysms, aneurysm volume reduction occurred in $88 \%(7 / 8)$ of aneurysms, and ischemic or hemorrhagic complications occurred in $0 \%(0 / 8)$ of patients. $19 \%(3 / 16)$ of patients that remained asymptomatic in the follow-up period and underwent cross-sectional imaging had clinically silent basal ganglia infarcts identified.

Conclusion Pipeline embolization of cerebral aneurysms in the M1 segment and middle cerebral artery bifurcation demonstrated a $71 \%$ rate of complete aneurysm occlusion. There were no deaths or disabling strokes, but there was an $8 \%$ rate of non-disabling ischemic strokes. Further work is necessary to describe the long-term effects of silent basal ganglia infarcts caused by PED.

Disclosures D. Lauzier: None. B. Root: None. Y. Kayan: 2; C; Microvention, Penumbra, Medtronic. J. Delgado Almandoz: 2; C; Medtronic, Microvention. J. Osbun: 2; C; Medtronic, Microvention. A. Chatterjee: None. K. Whaley: None. M. Tipps: None. C. Moran: 2; C; Medtronic, Cerenovus. A. Kansagra: 2; C; Penumbra, Microvention, iSchemaView.

\section{E-079 SEVERE, INTOLERABLE FATIGUE ASSOCIATED WITH A HYPER-RESPONSE TO CLOPIDOGREL}

${ }^{1} \mathrm{D}$ Bass ${ }^{*},{ }^{1} \mathrm{C}$ Young, ${ }^{2} \mathrm{M}$ Park, ${ }^{1} \mathrm{D}$ Bass, ${ }^{1} \mathrm{~K}$ Carroll, ${ }^{1} \mathrm{~K}$ Vanet, ${ }^{3} \mathrm{C}$ Lee, ${ }^{1} \mathrm{R}$ Sen, ${ }^{1} \mathrm{~S}$ Levy, ${ }^{1} \mathrm{C}$ Kelly, ${ }^{1} \mathrm{~L}$ Kim, ${ }^{1} \mathrm{M}$ Levitt. ${ }^{1}$ Neurological Surgery, University of Washington, Seattle, WA; ${ }^{2}$ Neurological Surgery, University of Virginia, Charlottesville, VA; ${ }^{3}$ School of Medicine, Washington State University, Spokane, WA

\subsection{6/neurintsurg-2021-SNIS.174}

Purpose and Background Clopidogrel is a commonly used antiplatelet for the prevention of thromboembolic complications following several types of neuroendovascular procedures. Its widespread use stems from its relatively low cost, good efficacy, and safety profile. However, concern has emerged for the possibility that clopidogrel may induce severe, intolerable fatigue in a small subset of patients. Thus, the purpose of this study is to systematically investigate this phenomenon and to better characterize the demographic at risk.

Methods We performed a dual-institution, 9-year, retrospective study of patients undergoing dual antiplatelet therapy with clopidogrel and aspirin for neuroendovascular procedures. Patients were included only if their response to clopidogrel was assessed by a VerifyNow P2Y12 (VNP) assay, which measures platelet inhibition using P2Y12 reactivity units (PRU). A hyper-response to clopidogrel is defined by a PRU $\leq 60$. Patients were considered to have had clopidogrel-induced severe fatigue if 1 ) the onset of severe, intolerable fatigue followed the initiation of clopidogrel, 2) fatigue resolved or improved following a reduction in the dose of clopidogrel, 3) no hemorrhagic event occurred while on clopidogrel, and 4) fatigue could not be attributed to any other medical explanation.

Results Clinical and demographic data were collected on 348 patients across 2 separate institutions. Five patients (1.4\%) were identified as having met criteria for clopidogrel-induced severe fatigue. All 5 patients were female, ages 39-68. In addition to severe fatigue, they suffered from headache, nausea, vertigo, and dyspnea. Each patient had been taking clopidogrel for at least one week prior to the onset of symptoms, and VNP assays obtained while the patients were experiencing their symptoms revealed hyper-responses to clopidogrel, with PRU between 0-22. Four of these patients initially had an appropriate response to clopidogrel, with a PRU > 60 immediately prior to the intervention, and they did not report fatigue until later. In response to the severe, intolerable fatigue, clopidogrel was reduced by half, resulting in resolution or improvement of symptoms in all 5 patients. However, VNP assays returned persistently low values (0-16 PRU). One patient developed anemia after her symptoms had already resolved, but no additional complications were reported during the course of their therapy. Notably, 30\% of patients $(n=103)$ demonstrated a hyper-response to clopidogrel on at least one VNP assay, but the vast majority of these patients did not suffer from severe fatigue. The rate of hyper-responses was similar across institutions and across genders.

Conclusions Severe, intolerable fatigue is a rare but clinically significant side effect of clopidogrel. It is associated with a hyper-response to therapy as determined by a PRU value $\leq 60$ on a VNP assay, but it appears that inhibition of platelet aggregation is merely epiphenomenal. Symptoms develop after an initial loading period and respond well to decreasing the dosage of clopidogrel. Only women were affected in our study, but this gender effect may reflect a sample bias as most of our patients were women.

Disclosures D. Bass: None. C. Young: None. M. Park: None. D. Bass: None. K. Carroll: None. K. Vanet: None. C. Lee: None. R. Sen: None. S. Levy: None. C. Kelly: None. L. Kim: None. M. Levitt: None.

\section{E-080 SWITCHING FROM TRANSFEMORAL TO TRANSRADIAL ACCESS DURING NEUROENDOVASCULAR PROCEDURES: A SINGLE-CENTER RETROSPECTIVE REVIEW OF OUTCOME AND COMPLICATIONS}

M Taqi ${ }^{*}$. Vascular Neurology of Southern California, Thousand Oaks, CA

\subsection{6/neurintsurg-2021-SNIS. 175}

Introduction/Purpose The transradial approach to endovascular therapy continues to gain traction as a comparable method of access to patient vasculature. Despite increasing the number of neurointerventionalist's utilizing the technique, questions regarding patient safety and outcome continue to exist among physicians.

Materials/Methods A retrospective review of patient charts was conducted from a single-center located in Thousand Oaks, California, USA treated from December 2018 to June 2020. The population of patients included in this analysis are those 